\title{
Cerebellar degeneration following neuroleptic malignant syndrome
}

\author{
V Lal, V Sardana, A Thussu, I M S Sawhney, S Prabhakar
}

\begin{abstract}
Summary
A 55-year-old woman with a history of bipolar affective disorder developed hyperpyrexia, rigidity and depressed consciousness (neuroleptic malignant syndrome) after commencing neuroleptic therapy. On regaining consciousness, she was mute and had signs suggesting pancerebellar involvement. Hyperpyrexia, which is a cardinal feature of neuroleptic malignant syndrome, may have caused cerebellar damage. Neuroleptic malignant syndrome needs both early recognition and prompt treatment to obviate devastating complications
\end{abstract}

Keywords: neuroleptic malignant syndrome, hyperpyrexia, cerebellar damage, mutism

Neuroleptic malignant syndrome is a catastrophic disorder characterised by the cardinal manifestations of intractable hyperthermia, generalised rigidity, depressed consciousness and autonomic dysfunction. Its estimated incidence ranges between $0.4 \%$ to $1.4 \%$ of cases treated with neuroleptics. ${ }^{1}$ The disorder typically develops within two weeks of commencing neuroleptic therapy but also occurs after prolonged treatment.

Neuroleptic malignant syndrome has a mortality rate of $20 \%$. Survivors recover rapidly and completely without long-term clinical sequelae. Cerebellar damage has been rarely documented after neuroleptic malignant syndrome, ${ }^{2}$ although it is not uncommon in other heat-related disorders. ${ }^{3}$ The rarity of this complication is highlighted in this case report.

\section{Case report}

A 50-year-old woman was admitted with altered sensorium, hyperpyrexia and generalised rigidity. She had maintained good health until four years earlier, when the onset of depressive symptoms necessitated treatment with the tricyclic antidepressant amitriptyline. She subsequently developed manic symptoms which responded to lithium and nitrazepam therapy. A recrudesence of her symptoms one week prior to admission led to the introduction of haloperiodol $5 \mathrm{mg}$ orally tid.

Four days later, she developed intractable hyperpyrexia and alteration in sensorium. A diagnosis of heat stroke was entertained and cold sponging and other adjunctive measures were commenced, to which there was no response. On further aggravation of her condition, she was transferred to our hospital.

On general physical examination, rectal temperature was $42^{\circ} \mathrm{C}$. Her pulse rate fluctuated widely between $130-250$ beats $/ \mathrm{min}$, with occasional ventricular and supraventricular ectopics. She had recurrent episodes of hypotension (systolic $70 \mathrm{mmHg}$ ) alternating with near normal/hypertensive values. She also exhibited marked hyperhidrosis which was most marked on the trunk and face. On neurologic examination she was profoundly comatose with bilaterally sluggish pupils of $2 \mathrm{~mm}$ diameter. She had generalised rigidity with hyperreflexia and upgoing plantars. The upper limbs showed dystonic posturing. Ancillary investigations disclosed persistent polymorphonuclear leucocytosis, markedly elevated creatine kinase levels $(2500 \mathrm{IU} / \mathrm{l})$ and hepatic and renal dysfunction. Blood gas studies revealed respiratory alkalosis. The cerebrospinal fluid was acellular with normal sugar and marginally elevated proteins $(80 \mathrm{mg} / \mathrm{l})$.

In light of the presence of the triad of generalised rigidity, hyperpyrexia and autonomic dysfunction, a tentative diagnosis of neuroleptic malignant syndrome was made. She was administered bromocriptine in doses ranging from $2.5 \mathrm{mg}$ to $10 \mathrm{mg}$ per day, on an incremental basis. Intravenous ciprofloxacin and metronidazole were administered for hospital-acquired bronchopneumonia. Parenteral dilantin was administered for generalised seizures, without any further recurrence.

With the above measures and adjunctive supportive care, her mental status ameliorated after two weeks, but new neurological signs surfaced. On regaining consciousness she was noted to be withdrawn and mute with normal comprehension. She also had ocular dysmetria and pronounced ataxia and tremor of limbs and the trunk, suggesting cerebellar dysfunction. Sensations were normal and plantars were flexor with near normal power. Three weeks later she started making rudimentary sounds and subsequently developed scanning dysarthria. Her cranial computed tomogram was normal.

On follow-up four months later, her signs and symptoms were unchanged. Her word output was marginally better than before, although it had the same scanning character.

\section{Discussion}

Our patient exhibited extreme hyperthermia, rigidity, autonomic dysfunction with profuse diaphoresis and elevated creatine kinase levels, 
all of which are the hallmarks of neuroleptic malignant syndrome. This developed soon after the introduction of haloperidol for her psychotic symptoms, a pattern which is in conformity with previously reported cases $^{1,2}$. We speculate that cerebellar damage resulted from intractable hyperthermia related to psychotropic medications.

Neuroleptics induce two distinct catastrophic disorders associated with hyperpyrexia, which differ from each other both clinically and pathophysiologically: neuroleptic malignant syndrome $^{2}$ and heat stroke ${ }^{4}$. Whereas the clinical picture of heat stroke is characterised by elevated body temperatures, and severe neurological dysfunction such as seizures, delirium or coma, associated with multiple organ dysfunction, it can be differentiated from neuroleptic malignant syndrome by the absence of extrapyramidal signs, absence of diaphoresis and a history of physical exercise or exposure to high ambient temperature ${ }^{1,3}$. Pathophysiologically, the genesis of hyperthermia in the two disorders is slightly different. Hyperthermia in neuroleptic malignant syndrome results from profound dopamine receptor blockage simultaneously in basal ganglia (leading to rigidity and enhanced thermogenesis) and in the hypothalamus (impairing heat dissipation and autonomic dysfunction). ${ }^{1,5}$ The genesis of hyperthermia in neuroleptic-induced heat stroke is dependent both on their anticholinergic and anti-alpha adrenergic attributes, which prevent heat dissipation by blocking sweating and enhance heat absorption from the environment, respectively. However, dopaminergic blockage of basal ganglia and hypothalamus is less pronounced in neuroleptic-induced heat stroke.

Neuronal function in the central and peripheral nervous system is significantly altered by even moderate changes in temperature, without causing structural injury to the nervous system. ${ }^{6}$ At extremes of temperature $\left(>42^{\circ} \mathrm{C}\right)$, such injury does occur. The cerebellum is most vulnerable to hyperthermia, followed by cerebral cortex, brainstem and spinal cord..$^{4,7}$ Purkinje cell loss occurs within 24 hours of hyperpyrexia and the extent of damage correlates with the degree and duration of hyperthermia. Mild cases show subtle cell changes consisting of neuronal swelling, pyknosis, or focal drop out, while profound cell loss accompanies severe cases. Consistent with this, the commonest neurological sequelae of hyperpyrexia include the syndrome of ataxia and dysarthria, persistent hemiparesis, seizures, myelopathy or quadriparesis. ${ }^{7,8}$

1 Addonizio G, Susman VI, Roth SD. Neuroleptic malignant syndrome: review of 115 cases. Biol Psychiatry 1987; 22: $1004-20$.

2 Lee S, Merriam A, Kim T-S, Liebling M, Dickson DW, Moore GRW. Cerebellar degeneration in neuroleptic malignant syndrome: neuropathological findings and review of the literature concerning heat-related nervous system injury. $\mathcal{F}$ Neurol Neurosurg Psychiatry 1989; 52: 387-91.

3 Yaqub BA, Daif AK, Papayiotopoulos CP. Pancerebellar syndrome in heat stroke: clinical course and CT scan findings. Neuroradiology 1987; 29: 294-6.

4 Lefkowitz D, Ford CS, Rich C, Biller J, McHenry Jr LC. Cerebellar syndrome following neuroleptic induced heat stroke. $\mathcal{F}$ Neurol Neurosurg Psychiatry 1983; 46: 183-5.

5 Buckley PF, Hutchinson M. Neuroleptic malignant syndrome. F Neurol Neurosurg Psychiatry 1995; 58: 271-3.

\section{Learning points}

- neuroleptic malignant syndrome is characterised by hyperpyrexia, rigidity, and autonomic dysfunction

- it is distinguished from neuroleptic-induced heat stroke by the presence of diaphoresis, rigidity and lack of history of physical exertion

- both neuroleptic malignant syndrome and heat stroke can lead to residual cerebellar damage due to hyperpyrexia

- the cerebellum is most vulnerable to hyperpyrexia and cerebellar dysfunction occurs frequently in heat-induced central nervous system injury.

To our knowledge cerebellar damage following neuroleptic malignant syndrome has been described only once in the literature by Lee $e t a l^{2}$, although it has been described frequently in heat-induced central nervous system (CNS) injury, due to heat stroke, ${ }^{3}$ and fever therapy. ${ }^{8}$ In the only autopsy study of cerebellar damage induced by neuroleptic malignant syndrome available in the literature, ${ }^{2}$ neuronal loss was most prominant in the cerebellum but not in the cerebral cortex, basal ganglia or hippocampus, indicating selective vulnerability of cerebellar neurons to heat-induced injury. This is in contrast to hypoxic CNS injury which has a predilection for involving different regions of the brain including cerebral cortex, hippocampus, cerebellum and basal ganglia. ${ }^{9}$

On regaining consciousness, the patient was mute for a protracted period. Transient muteness has been documented in acute bilateral cerebellar injury. ${ }^{10}$ The cerebellum functions primarily to modify the force and rapidity of muscular activity resulting in finely coordinated muscular activity, including speech. Devastating cerebellar dysfunction may result in the most profound derangement of speech function, mutism. ${ }^{10}$

The mortality and morbidity of neuroleptic malignant syndrome has declined considerably during the past decade due to early recognition and improved management. The most effective measures, besides prompt recognition, include withdrawal of neuroleptic medication and transfer to an intensive care unit, with attention to hydration, fever reduction, sedation and control of rigidity with bromocriptine or dantrolene. ${ }^{5}$ The above case underscores the importance of early recognition and prompt treatment to prevent long term sequelae.

6 Botton CF, Sawa GM, Carter K. The effect of temperature on human compound action potentials. $\mathcal{F}$ Neurol Neurosurg Psychiatry 1981; 44: 407-13.

7 Delgado G, Tunon T, Gallego J, Villanereva JA. Spinal cord lesions in heat stroke. $\mathcal{f}$ Neurol Neurosurg Psychiatry 1985; 48: $1065-7$.

8 Gore I, Issacson NH. The pathology of hyperpyrexia: observation at autopsy in 17 cases of fever therapy. $\mathrm{Am}$ Pathol 1949; 25: 1029-59.

9 Longstreth WT Jr. Neurological complications of cardiac arrest. In: Aminoff MJ, ed, Neurology and general medicine, 2nd edn. Edinburgh: Churchill Livingstone, 1996; pp 15982.

10 Rekate HL, Grubb RL, Aram DM, Hahn JF, Ratcheson RA. Mutism of cerebellar origin. Arch Neurol 1985; 42:
R97-8. 